\title{
A NOTE ON LOGARITHMS OF NORMAL OPERATORS ${ }^{1}$
}

\section{SVETOZAR KUREPA}

All operators considered in this note are bounded and defined on a fixed Hilbert space $X$. In [4], C. R. Putnam has proved that if $H$ is a positive definite selfadjoint operator and $\exp T=H$, then $\|T\|$ $\leqq 2 \ln 2$ implies that $T$ is a selfadjoint operator. In Theorem 3 we prove that it is sufficient to assume that $\|T\|<2 \pi$ in order that $T$ be selfadjoint. This condition, already in the set of complex numbers, cannot be replaced by $\|T\| \leqq 2 \pi$ without changing the conclusion. In Theorem 4 we prove that $\|T\| \leqq 2 \pi$ in a finite dimensional space implies that $T$ is a normal operator. In Theorem 2 some conditions for a logarithm $T$ of a normal operator $N$ are derived. Assuming that the spectrum of $N$ lies in the set

$$
\Omega=\left\{r e^{i \phi} \mid-\alpha \pi \leqq \phi \leqq \alpha \pi, 0 \leqq \alpha \leqq \frac{1}{2}, r \geqq \epsilon>0\right\}
$$

we prove that $\exp T=N$ and $\|T\|<\left(1-\alpha^{2} / 4\right) \pi$ imply that $T$ is a normal operator. All these results are consequences of

THEOREM 1. Let $X$ be a Hilbert space and $T: X \rightarrow X$ a bounded operator such that $\exp T=N$ is a normal operator. Then

$$
T=N_{0}+2 \pi i W
$$

with

$$
N_{0}=\int \ln \lambda d E(\lambda)
$$

where $\ln \lambda$ is the principal (or any) branch of the logarithmic function and $E(\lambda)$ is the spectral measure of $N$. The bounded operator $W$ commutes with $N_{0}$ and there exists a bounded and regular, positive definite selfadjoint operator $Q$ such that

$$
W_{0}=Q^{-1} W Q
$$

is a selfadjoint operator the spectrum of which belongs to the set of all integers ( $c f .[2$, Theorem 4; 3, Lemma 2.2 and Theorem III]).

Proof. The operator $N_{0}$ defined by (3) is bounded and $\exp N_{0}=N$.

Presented to the Society, February 25, 1961; received by the editors January 26 , 1961 and, in revised form, March 20, 1961.

1 This work was supported by the National Science Foundation NSFG 9423. 
Since $T$ commutes with $N$, it commutes with $E(\lambda)[1$, p. 68, Theorem 2] and therefore $T$ commutes also with $N_{0}$. Thus the operator $2 \pi i W=T-N_{0}$ has the property that $\exp 2 \pi i W=E$, where $E$ is the identity operator. Now, the one-parameter group

$$
G(t)=\exp 2 \pi i t W
$$

with $t \in R$ ( $R$ denotes the set of all real numbers), is continuous and periodic. Thus

$$
\sup _{t \in R}\|G(t)\|<+\infty
$$

which, by the well-known theorem of B. Sz. Nagy [5], implies the existence of a regular, positive definite, selfadjoint operator $Q$ such that

$$
Q^{-1} G(t) Q=\exp 2 \pi i t Q^{-1} W Q
$$

is a group of unitary operators. But this is possible if and only if the operator

$$
W_{0}=Q^{-1} W Q
$$

is selfadjoint. Now $\exp 2 \pi i W_{0}=E$ and the fact that $W_{0}$ is selfadjoint imply that the spectrum of $W_{0}$ consists of integers only. Q.E.D.

Theorem 2. Let $X$ be a Hilbert space, $N: X \rightarrow X$ a bounded normal operator the spectrum of which is contained in the set (1). If $T: X \rightarrow X$ is a bounded operator such that

$$
\exp T=N \text { and }\|T\|<\left(1-\frac{\alpha^{2}}{4}\right) \pi,
$$

then $T$ is a normal operator.

Proof. Let $E(\lambda)$ be the spectral measure of $N$ and

$$
N_{0}=\int_{\Omega} \ln \lambda d E(\lambda)
$$

where $\ln \lambda=\ln |\lambda|+i \arg \lambda,-\pi \leqq \arg \lambda<\pi$. According to Theorem 1 we have $T=N_{0}+2 \pi i W$. We will prove that $W=0$. First of all we have:

$$
\max \left(\|N\|,\left\|N^{-1}\right\|\right)<\exp \left(1-\frac{\alpha^{2}}{4}\right) \pi .
$$

This, the definition of $N_{0}$, and 


$$
\left\|N_{0}\right\|=\sup _{\lambda \in \sigma(N)}|\ln \lambda|<\left[\alpha^{2} \pi^{2}+\left(1-\frac{\alpha^{2}}{4}\right)^{2} \pi^{2}\right]^{1 / 2},
$$

where $\sigma(N)$ denotes the spectrum of $N[1$, p. 62, Theorem 2], imply:

$$
\begin{aligned}
2 \pi \mid W \| & \leqq\|T\|+\left\|N_{0}\right\|<\pi\left(1-\frac{\alpha^{2}}{4}\right)+\pi\left[\alpha^{2}+\left(1-\frac{\alpha^{2}}{4}\right)^{2}\right]^{1 / 2} \\
& =2 \pi .
\end{aligned}
$$

Thus

$$
\|W\|<1 .
$$

On the other hand $W_{0}=Q^{-1} W Q$, where $Q$ and $W_{0}$ are selfadjoint operators and the spectrum of $W_{0}$ is contained in the set of all integers. If $W_{0} x=n x$, then $W y=n y$, with $y=Q x /\|Q x\|$. But then $\|W\| \geqq|n|$ which by virtue of (7) gives $n=0$. Thus the spectrum of $W_{0}$ consists of the origin only. This implies $W_{0}=0$ and therefore $W=0$ which implies $T=N_{0}$. Q.E.D.

REMARK 1. In a two dimensional unitary space the operator $T$, which in an orthonormal basic set has the matrix

$$
i\left(\begin{array}{cc}
1 & 0 \\
1 & 1-2 \pi
\end{array}\right)
$$

possesses the following properties: (a) $T$ is not normal, (b) exp $T$ $=E e^{i}$ and (c) $\|T\|<2 \pi$. This example shows that in general the normality of $N=\exp T$ and $\|T\|<2 \pi$ does not imply that $T$ is a normal operator. But in the case in which $N$ is a positive definite, selfadjoint operator this is true, because we have:

Theorem 3. Let $X$ be a Hilbert space, $H: X \rightarrow X$ a bounded, positive definite, selfadjoint operator and $T: X \rightarrow X$ an operator such that

$$
\exp T=H \text { and }\|T\|<2 \pi \text {. }
$$

Then the operator $T$ is a selfadjoint operator.

Proof. The operator $H_{0}=N_{0}$ defined by (3), with $\ln \lambda$ as the principal branch of the logarithm, is a bounded selfadjoint operator and $T=H_{0}+2 \pi i W$. Again, if $x \neq 0, W_{0} x=Q^{-1} W Q x=n x$, then $W y=n y$, with $y=Q x /\|Q x\|$, implies

$$
(T y, y)=\left(H_{0} y, y\right)+2 \pi i n .
$$

Since $H_{0}$ is selfadjoint, $\left(H_{0} y, y\right)$ is real and we get:

$$
|(T y, y)|^{2}=\left(H_{0} y, y\right)^{2}+4 \pi^{2} n^{2} \leqq\|T\|^{2}<4 \pi^{2} .
$$


Thus $n^{2}<1$, which implies $n=0$. This obviously leads to $W=0$ and therefore $T=H_{0}$. Q.E.D.

Corollary 1. If $T$ is a bounded linear operator and $H$ a positive definite, selfadjoint operator such that

$$
\exp T=H e^{i \theta},
$$

with $\theta \in[0,2 \pi]$, then

$$
\|T\| \geqq \theta \text { if } \theta \in[0, \pi] \text { and }\|T\| \geqq \pi-\theta \text { if } \theta \in[\pi, 2 \pi] .
$$

Proof. Suppose that $\theta \in[0, \pi]$ and $\|T\|<\theta$. Then $\|T-i \theta E\|<2 \pi$ and $\exp (T-i \theta E)=H$, together with Theorem 3, imply that $H_{0}=T$ $-i \theta E$ is a selfadjoint operator. But then $|(T x, x)|^{2}=\left(H_{0} x, x\right)^{2}+\theta^{2}$ $\leqq\|T\|^{2}<\theta^{2}$ holds for any $x \in X,\|x\|=1$. Since this is impossible, we have $\|T\| \geqq \theta$. If $\theta \in[\theta, 2 \pi]$, then $2 \pi-\theta \in[0, \pi]$ and we have $\|T\|$ $=\left\|T^{*}\right\| \geqq 2 \pi-\theta$ because of $\exp T^{*}=H e^{i(2 \pi-\theta)}$.

Theorem 4. Let $X$ be a finite dimensional unitary space, $H: X \rightarrow X$ a positive definite, selfadjoint operator and $T: X \rightarrow X$ an operator such that

$$
\exp T=H \quad \text { and } \quad\|T\| \leqq 2 \pi .
$$

Then $T$ is a normal operator.

Proof. Since $H$ is selfadjoint, we have

$$
H=\sum_{k=1}^{m} \lambda_{k} E_{k}
$$

where $\lambda_{k}$ are positive numbers, $\lambda_{k} \neq \lambda_{k^{\prime}}$ if $k \neq k^{\prime}$, and $E_{k}$ are selfadjoint pairwise orthogonal projections. If $X_{k}$ denotes the range of $E_{k}$, then $X$ is an orthogonal sum of the subspaces $X_{k}$. Since $T$ commutes with $H$, we have

$$
T=\sum_{k=1}^{m} \oplus T_{k},
$$

where $T_{k}$ is the restriction of $T$ to $X_{k}$ and the symbol $\oplus$ denotes the orthogonal sum of operators. Now (8) becomes:

$$
\exp T_{k}=\lambda_{k} E_{k}, \quad\left\|T_{k}\right\| \leqq 2 \pi, \quad k=1,2, \cdots, m .
$$

If we prove that every $T_{k}$ is normal, then $T$ as the orthogonal sum of normal operators will be normal.

Therefore it is sufficient to prove, that if $X$ is an $n$-dimensional unitary space, $\exp T=\lambda E, \lambda>0$ and $\|T\| \leqq 2 \pi$, then $T$ is normal. According to Theorem 1 we have $T=E \ln \lambda+2 \pi i W$ with $\ln \lambda$ real for 
$\lambda>0$. Now, $W y=n y,\|y\|=1$, implies $T y=\ln y+2 \pi i n y$ and therefore

$$
\|T y\|^{2}=(\ln \lambda)^{2}+4 \pi^{2} n^{2} \leqq 4 \pi^{2} .
$$

If $\ln \lambda \neq 0$, then $n=0$ and $T=E \ln \lambda$, i.e. $T$ is selfadjoint. On the other hand, if $\ln \lambda=0$, then $T=2 \pi i W$, the spectrum of $W$ is contained in the set $\{-1,0,1\}$ and $\|W\| \leqq 1$. To prove that $W$ is normal we take an orthonormal basic set $e_{1}, \cdots, e_{n}$ in $X$ such that the matrix $W(e)$ which belongs to $W$ in this basic set has the property that $[W(e)]_{i j}$ $=0$ if $1 \leqq i<j \leqq n$. Now

$$
\left\|W e_{1}\right\|^{2}=\left|[W(e)]_{11}\right|^{2}+\left|[W(e)]_{21}\right|^{2}+\cdots+\left|[W(e)]_{n 1}\right|^{2} \leqq 1 .
$$

Hence, if $\left|[W(e)]_{11}\right|=1$, then $[W(e)]_{21}=\cdots=[W(e)]_{n 1}=0$. This method implies that the matrix $W(e)$ has the following form:

$$
W(e)=\left(\begin{array}{cc}
W_{0}(e) & 0 \\
0 & W_{1}(e)
\end{array}\right),
$$

where $W_{0}(e)$ is a diagonal matrix and all elements of the matrix $W_{1}(e)$ on and above the main diagonal are zero. Obviously exp $2 \pi i W_{1}(e)$ $=E_{1}(e)$, where $E_{1}(e)$ is the unit matrix of the same order as $W_{1}(e)$. Since $W_{1}(e)$ has all eigenvalues zero, and since it is similar to a hermitian matrix (Theorem 1), we find $W_{1}(e)=0$. Thus the matrix $W(e)$ is diagonal, i.e., the operator $W$ is a normal operator. Q.E.D.

REMARK 2. In a two-dimensional unitary space, the operator $T$, which in an orthonormal basic set has the matrix

$$
2 \pi i\left(\begin{array}{cc}
1 & 0 \\
\epsilon & 0
\end{array}\right), \quad \epsilon \neq 0
$$

has the following properties: (1) $T$ is not normal, (2) $\exp T=E$ and (3) $\|T\|=2 \pi\left(1+\epsilon^{2}\right)^{1 / 2}$, i.e., the condition $\|T\| \leqq 2 \pi$ of Theorem 4 cannot be weakened without altering the conclusion.

\section{REFERENCES}

1. P. R. Halmos, Introduction to Hilbert space and the theory of spectral multiplicities, Chelsea, New York, 1951.

2. E. Hille, On roots and logarithms of elements of a complex Banach algebra, Math. Ann. 136 (1958), 46-57.

3. G. L. Krabbe, On the logarithm of a uniformly bounded operator, Trans. Amer. Math. Soc. 81 (1956), 155-166.

4. C. R. Putnam, On square roots and logarithms of self-adjoint operators, Proc. Glasgow Math. Assoc. 4 (1958), 1-2.

5. B. Sz. Nagy, On uniformly bounded linear transformations in Hilbert space, Acta Sci. Math. Szeged 11 (1947), 152-157.

Department of Mathematics, Zagreb, Yugoslavia and UNIVERSITY OF MARYLAND 